\title{
Synchronization in Nonidentical Extended Systems
}

\author{
S. Boccaletti, ${ }^{1}$ J. Bragard, ${ }^{2,3}$ F. T. Arecchi, ${ }^{2,4}$ and H. Mancini ${ }^{1}$ \\ ${ }^{1}$ Department of Physics and Applied Mathematics, Universidad de Navarra, Irunlarrea s/n, 31080 Pamplona, Spain \\ ${ }^{2}$ Istituto Nazionale di Ottica, Largo E. Fermi, 6, I50125 Florence, Italy \\ ${ }^{3}$ Department of Physics, University of Liege, Liege, Belgium \\ ${ }^{4}$ Department of Physics, University of Florence, Florence, Italy
}

(Received 25 March 1999)

\begin{abstract}
We report the synchronization of two nonidentical spatially extended fields, ruled by one-dimensional complex Ginzburg-Landau equations, both in the phase and in the amplitude turbulence regimes. In the case of small parameter mismatches, the coupling induces a transition to a completely synchronized state. For large parameter mismatches, the transition is mediated by phase synchronization. In the former case, the synchronized state is not qualitatively different from the unsynchronized one, while in the latter case the synchronized state may substantially differ from the unsynchronized one, and it is mainly dictated by the synchronization process of the space-time defects.
\end{abstract}

PACS numbers: 05.45.Xt, 05.45.Pq, 05.45.Jn, 47.54.+r

Coupled chaotic concentrated systems may display four degrees of synchronization, namely, complete synchronization (CS) [1], phase synchronization (PS) [2], lag synchronization (LS) [3], and generalized synchronization (GS) [4]. In CS, a perfect hooking of the chaotic trajectories of two systems is achieved by means of a coupling signal, in such a way that they remain in step with each other in the course of time. This mechanism has been shown to occur when two identical chaotic systems are coupled, provided that all the sub-Liapunov exponents of the subsystem to be synchronized are negative [1]. Nonidentical systems can reach a regime (PS), wherein a locking of the phases is produced, while the amplitudes remain uncorrelated [2]. The transition to PS for two coupled oscillators has been characterized with reference to the Rössler system [3,5]. LS is an intermediate step between PS and CS. In this case, the two signals lock their phases and amplitudes, but with a time lag, $s_{1}(t) \simeq s_{2}\left(t-\tau_{\text {lag }}\right)$ [3]. Finally, GS consists in the hooking of the output of one system to a given function of the output of the other system [4].

The generic scenario for concentrated symmetrically coupled nonidentical systems consists in successive transitions between PS, LS, and CS when increasing the coupling strength [3].

When passing to space-extended systems, space-time chaos synchronization has been studied in discrete systems, such as populations of coupled dynamical systems [6], systems formed by globally coupled Hamiltonian [7] or bistable elements [8], and neural networks [9]. As for continuous systems, the emergence of complete synchronized states has been studied for one-dimensional chemical models [10], and for two fields obeying the identical one-dimensional complex Ginzburg-Landau (CGL) equation [11].

In this Letter we characterize the emergence of synchronized behaviors in continuous nonidentical spaceextended symmetrically coupled pattern forming systems.
For the sake of exemplification, and without significant loss of generality, we will refer to a pair of onedimensional fields $A_{1,2}(x, t)$ evolving in space and time following the CGL equation. This equation describes the universal pattern forming features close to the emergence of a Hopf bifurcation [12]. It has been used to describe many different situations in laser physics, fluid dynamics, chemical turbulence, bluff body wakes, etc. The system under study is

$$
\begin{aligned}
\dot{A}_{1,2}= & A_{1,2}+\left(1+i \alpha_{1,2}\right) \partial_{x}^{2} A_{1,2} \\
& -\left(1+i \beta_{1,2}\right)\left|A_{1,2}\right|^{2} A_{1,2}+\varepsilon\left(A_{2,1}-A_{1,2}\right),
\end{aligned}
$$

where $A_{1,2}(x, t) \equiv \rho_{1,2}(x, t) \exp \left[i \psi_{1,2}(x, t)\right]$ are two complex fields of amplitudes $\rho_{1,2}$ and phases $\psi_{1,2}$, respectively, $\partial_{x}^{2} A_{1,2}$ stays for the second derivative of $A_{1,2}$ with respect to the space variable $0 \leq x \leq L, L$ represents the system size, dot denotes temporal derivative, the control parameters $\alpha_{1,2}, \beta_{1,2}$ are real numbers, and $\varepsilon$ is the strength of the symmetric coupling. The boundary conditions are chosen to be periodic.

For $\varepsilon=0$, Eq. (1) describes two uncoupled fields $A_{1,2}$ each one obeying a separate CGL. This equation has plane wave solutions $A_{q}=\sqrt{1-q^{2}} e^{i(q x+\omega t)}$ where $-1 \leq q \leq 1$, with $q$ being the wave number in the Fourier space, and $\omega=-\beta-(\alpha-\beta) q^{2}$. Such solutions become unstable in the parameter region $\alpha \beta>-1$ outside the range $-q_{c} \leq q \leq q_{c}\left(q_{c}=\sqrt{\frac{1+\alpha \beta}{2\left(1+\beta^{2}\right)+1+\alpha \beta}}\right)$ through the so-called Eckhaus instability. Since $q_{c}$ vanishes as the product $\alpha \beta$ approaches -1 , all plane waves become unstable when crossing from below the $\alpha \beta=-1$ line in the parameter space, which is called Benjamin-Feir or Newell line. Above this line, Ref. [13] identifies three different turbulent states, namely, phase turbulence (PT), amplitude or defect turbulence (AT), and bichaos. We will specialize our analysis on PT and AT, since they have received special attention in the scientific community [14]. 
PT occurs just above the $\alpha \beta=-1$ line, and is characterized by a chaotic phase $\psi$, whereas the amplitude $\rho$ remains approximately constant. By further moving away from the $\alpha \beta=-1$ line, a transition is encountered toward AT, wherein the amplitude dynamics becomes dominant, leading to large amplitude oscillations which can occasionally drive $\rho$ to zero. The vanishing of $\rho$ causes the occurrence of a space-time defect.

In the following we will discuss the effect of $\varepsilon \neq 0$ in Eq. (1). GS was shown to hold for two identical extended fields in a particular dynamical situation [11], in which two identical CGL's (with $\alpha, \beta$ below the BenjaminFeir line) are coupled by an extra cubic term. Here we deal with nonidentical systems $\left(\alpha_{1} \neq \alpha_{2}, \beta_{1} \neq \beta_{2}\right)$, and consider both the case of small and large parameter mismatches. In the former case, the systems are prepared in the same dynamical regime, e.g., both in PT or in AT. In the latter case, the parameters $\alpha_{1}, \alpha_{2}, \beta_{1}, \beta_{2}$ are chosen so that one system is in the PT regime, while the other is in the AT regime.

We first consider small parameter mismatches, and select $\alpha_{1}=\alpha_{2}=2.1, \beta_{1}=-1.25$, and $\beta_{2}=-1.2$ in Eq. (1) (both fields in AT). Figure 1 reports the space-time plots of $\rho_{1}$ [Figs. 1(a), 1(c), 1(e)] and $\rho_{2}$ [Figs. 1(b), 1(d), 1 (f)] for $\varepsilon=0.05$ [Figs. 1(a), 1(b)], $\varepsilon=0.09$ [Figs. 1(c), 1(d)], and $\varepsilon=0.15$ [Fig. 1(e), 1(f)]. The moduli are coded into a 256 gray levels scale. The dark lines trace the positions of the space-time defects. The simulations have been performed with $L=64$, periodic boundary conditions, random initial conditions. The numerical code is based on a semi-implicit scheme in time with finite differences in space. The precision of the code is first order in time and second order in space. A space discretization $\delta x=0.125$ (512 mesh points) and an integration time step $\delta t=0.001$ have been used. Figure 1 shows a gradual passage from a nonsynchronized AT state [Figs. 1(a), 1(b)] to a completely synchronized AT state [Figs. 1(e), 1(f)], through an intermediate state [Figs. 1(c), 1(d)] wherein only partial synchronization is built. Notice that here the synchronization of the global structure implies the synchronization of each localized space-time defect [as it appears evident from Figs. 1(e), 1(f)].

At variance with what happens in concentrated systems, the transition from no synchronization to CS seems here not associated with the presence of an intermediate PS regime. This feature is confirmed by the measurements of $\Delta \rho=\left\langle\left|\rho_{1}-\rho_{2}\right|\right\rangle$ and $\Delta \psi=\left\langle\left|\psi_{1}-\psi_{2}\right|\right\rangle$ as functions of $\varepsilon$. Here $\langle\cdots\rangle$ stays for an averaging both in time and space. As it can be seen in Fig. 2, $\langle\Delta \rho\rangle$ and $\langle\Delta \psi\rangle$ both show a smooth decreasing behavior as functions of $\varepsilon$, thus meaning that amplitude and phase synchronization processes occur at the same time. The scenario is therefore consistent with what is already observed for small parameter mismatches in chemical models [10].

The same qualitative features occur when the parameters are selected so as both fields are in PT. Here again,
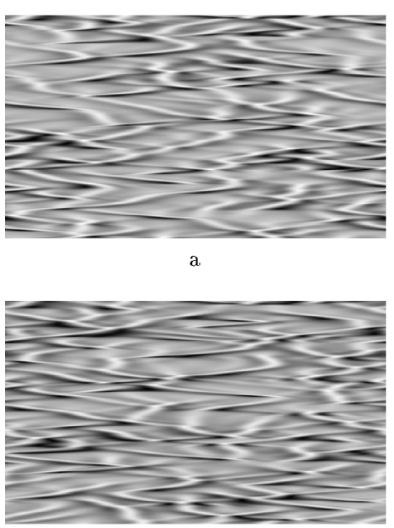

c
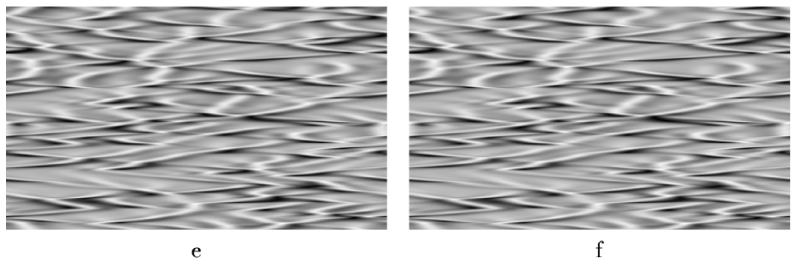

FIG. 1. AT-AT case: Space- (horizontal) time (vertical) plots of the moduli $\rho_{1}[(\mathrm{a}),(\mathrm{c}),(\mathrm{e})]$ and $\rho_{2}[(\mathrm{~b}),(\mathrm{d}),(\mathrm{f})]$. $\alpha_{1}=\alpha_{2}=2.1, \beta_{1}=-1.25$, and $\beta_{2}=-1.2$. Time increases downwards from 300 to 600 (u.t.). The first 300 time units (not plotted) correspond to the transient before the system reaches two independent chaotic (AT) states starting from two independent random initial conditions (the same remarks hold for Fig. 3). (a) and (b) correspond to $\varepsilon=0.05$, (c) and (d) to $\varepsilon=0.09$, and (e) and (f) to $\varepsilon=0.15$.

the system passes from an unsynchronized PT state at small coupling to a completely synchronized PT state. In this case, since defects are not present, the synchronization takes place at smaller coupling strengths.

A more interesting scenario emerges in the case of large parameter mismatches. Let us select in Eq. (1) $\alpha_{1}=\alpha_{2}=2.1, \beta_{1}=-1.2$, and $\beta_{2}=-0.83$, so as the field $A_{1}$ is evolving in AT, while the field $A_{2}$ is evolving in PT. In Fig. 3 we show the space-time representation of the patterns of $\rho_{1}$ [Figs. 3(a), 3(c), 3(e)] and $\rho_{2}$ [Figs. 3(b), 3(d), 3(f)] for $\varepsilon=0.09$ [Figs. 3(a), 3(b)], $\varepsilon=0.14$ [Figs. 3(c), 3(d)], and $\varepsilon=0.19$ [Figs. 3(e), 3(f)]. For small coupling strengths, the two systems do not synchronize, and they hold in their respective regimes [Figs. 3(a), 3(b)]. At large coupling strengths, the two systems reach a CS regime, which is realized in PT [Figs. 3(e), 3(f)]. This means that the final synchronized state is space-time chaotic, but the complete synchronization process is here associated with the suppression of all defects, which were initially present in the field $A_{1}$. In other words, since CS implies amplitude synchronization, the small amplitude oscillations of $A_{2}$ attract the synchronized set, thus suppressing the defects originally existing in the dynamics of $A_{1}$. However, the most interesting regime is the intermediate regime [Figs. 3(c), 3(f)], wherein the two systems partially synchronize, and they both recover an AT regime. Figure 4 reports the plots of 


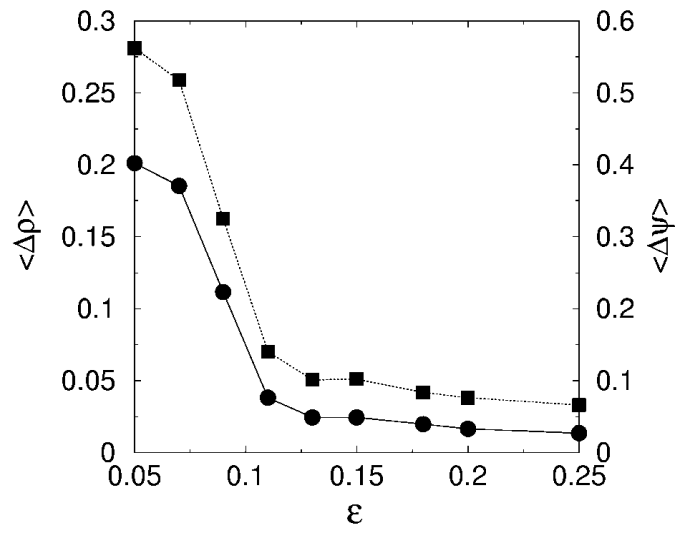

FIG. 2. AT-AT case: $\langle\Delta \rho\rangle,\langle\Delta \psi\rangle$ vs $\varepsilon$ (see definitions in the text). Same parameters as in the caption of Fig. 1. The left (right) vertical axis reports the $\langle\Delta \rho\rangle(\langle\Delta \psi\rangle)$ scale. Notice that $\langle\Delta \rho\rangle$ never vanishes completely, due to the parameter difference in the evolution equations for the two fields.

$\langle\Delta \rho\rangle$ and $\langle\Delta \psi\rangle$ as functions of $\varepsilon$. At variance with Fig. 2, here a quite wide range of $\varepsilon$ exists $(0.1 \leq \varepsilon \leq 0.16)$, wherein amplitude synchronization is not yet reached, while the global phase distance converges to a constant value, thus meaning the emergence of an intermediate PS state. In this state, the amplitudes of the two fields are still uncorrelated, while the phases are strongly coupled.

Now, since the natural evolution of $A_{1}$ is in AT, it shows the presence of many space-time defects. As discussed above, defects are localized points resulting by the vanishing of $\rho$. Therefore, in each one of them, the phase $\psi_{1}$ shows a singularity. One can imagine that AT
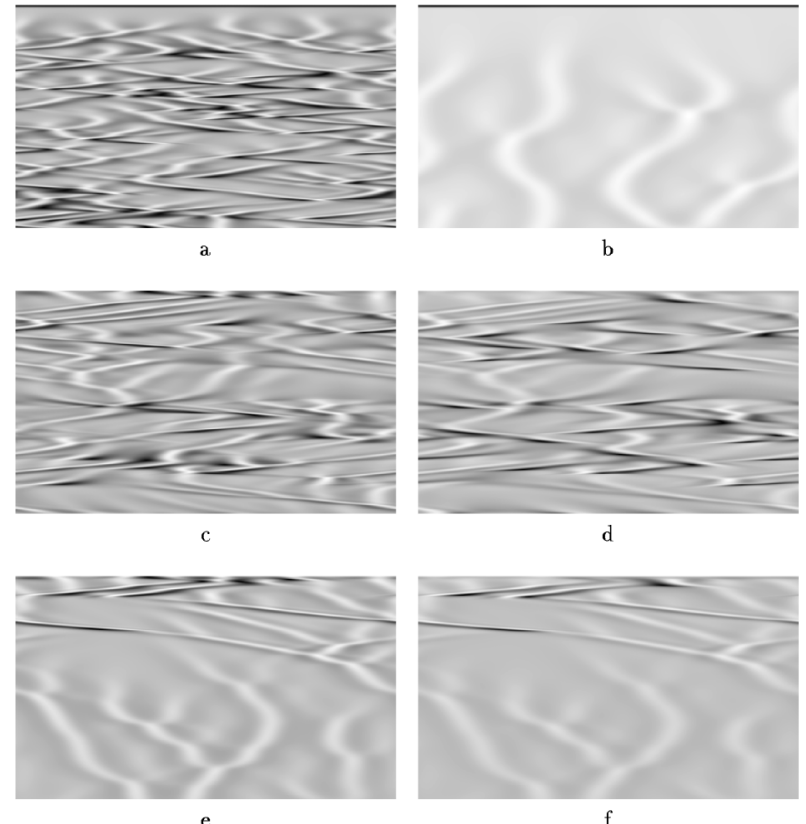

FIG. 3. AT-PT case: Space- (horizontal) time (vertical) plots of the moduli $\rho_{1}$ [(a), (c), (e)] and $\rho_{2}$ [(b), (d), (f)]. $\alpha_{1}=\alpha_{2}=2.1, \beta_{1}=-1.2$, and $\beta_{2}=-0.83$. (a) and (b) correspond to $\varepsilon=0.09$, (c) and (d) to $\varepsilon=0.14$, and (e) and (f) to $\varepsilon=0.19$. allows flexibility in the dynamics of the amplitude, but the variations of the phase are not flexible, and they are substantially determined by the local amplitude variations. On the contrary, $A_{2}$ would naturally evolve in PT, that is with a dominant phase dynamics. The phase $\psi_{2}$ is not naturally bounded, and its oscillations are allowed by the evolution of the system in the uncoupled case. In the range $0.1 \leq \varepsilon \leq 0.16$, a PS state is built. There, the phases $\psi_{1}$ and $\psi_{2}$ must converge (apart from a constant). This is possible only when the phase $\psi_{2}$ locally adjusts on $\psi_{1}$. The relevant consequence of this process is the introduction of many phase defects in the field $A_{2}$ [Fig. 3d], which would be instead free of them in the uncoupled state.

The above qualitative picture is quantitatively confirmed by the measurement of the total number of phase defects $N_{d}$ as a function of $\varepsilon$ for $\alpha_{1}=\alpha_{2}=2.1, \beta_{1}=$ -1.2 , and $\beta_{2}=-0.83$. Figure 5 reports $N_{d}$ vs $\varepsilon$ for $A_{1}$ (circles) and $A_{2}$ (squares). For small coupling strength $(0<\varepsilon<0.1)$ the two fields evolve in an unsynchronized manner [see Figs. 3(a), 3(b)]. At intermediate $\varepsilon$ values $(0.1<\varepsilon<0.16)$ there is a process of defect injection into the field $A_{2}$ up to the point $(\varepsilon \simeq 0.16)$ where both fields show the same defect number. Notice that this $\varepsilon$ range coincides exactly with the phase plateau in Fig. 4. The spectral analysis of the PS state reveals that, while the mean wave number is vanishing for both fields independently on $\varepsilon$, the mean frequencies are always bounded away from zero and show an interesting dynamics as functions of $\varepsilon$. Precisely, while $A_{1}$ appears to be robust in its frequency variations, $A_{2}$ shows large frequency variations as a function of $\varepsilon$, thus confirming our heuristic argument about the flexibility of $A_{2}$ during the synchronization process. A detailed analysis of this synchronization state will appear elsewhere.

When all defects have been synchronized, then the system begins to reach a CS state, which is realized in

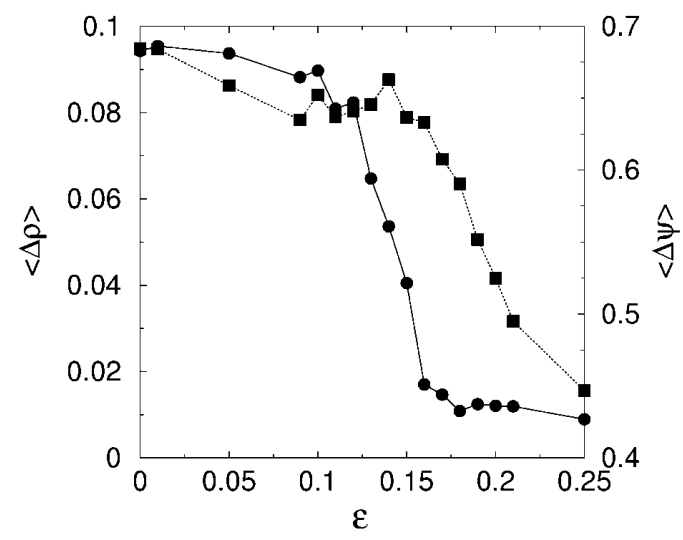

FIG. 4. AT-PT case: Indicators of modulus (circles) and phase (squares) synchronization (same stipulations as in Fig. 2). Same parameters as in the caption of Fig. 3. Note the phase plateau for $0.1 \leq \varepsilon \leq 0.16$. 


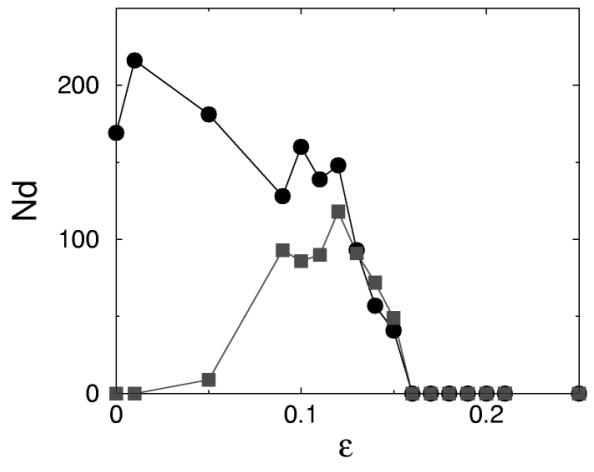

FIG. 5. AT-PT case: Total number of phase defects $N_{d}$ as a function of the coupling strength $\varepsilon$ for the fields $A_{1}$ (circles) and $A_{2}$ (squares). Same parameters as in the caption of Fig. 3.

PT [see Figs. 3(e), 3(f)], implying the absence of phase defects in both fields.

All the above scenarios are generally observed in Eq. (1) regardless of the particular choices of $\alpha_{1}, \alpha_{2}, \beta_{1}, \beta_{2}$ for suitable values of the coupling parameter $\varepsilon$.

In conclusion we have shown how CS and PS are built in nonidentical space-extended systems. In the case of small parameter mismatches, one observes a passage from nonsynchronized to a completely synchronized state. For large parameter mismatches, this transition is mediated by phase synchronization. While in the former case, the resulting space-time synchronized state is not qualitatively different from the unsynchronized one, in the latter case, the state of the system resulting from the synchronization process may substantially differ from that present with no coupling, and it is mainly dictated by the synchronization process of the space-time defects.

We acknowledge J. Kurths and A. Pikovski for useful discussions. This work was partly supported by Integrated Action Italy-Spain HI97-30. S. B. acknowledges financial support from EU Contract No. ERBFMBICT983466. J.B. benefits from a EU Network grant under Contract No. FMRXCT960010 "Nonlinear dynamics and statistical physics of spatially extended systems."

[1] L. M. Pecora and T.L. Carroll, Phys. Rev. Lett. 64, 821 (1990).

[2] M. G. Rosenblum, A.S. Pikovsky, and J. Kurths, Phys. Rev. Lett. 76, 1804 (1996).

[3] M. G. Rosenblum, A. S. Pikovsky, and J. Kurths, Phys. Rev. Lett. 78, 4193 (1997).

[4] L. Kocarev and U. Parlitz, Phys. Rev. Lett. 76, 1816 (1996).

[5] E. Rosa, Jr., E. Ott, and M. H. Hess, Phys. Rev. Lett. 80, 1642 (1998).

[6] S. H. Strogatz, R.E. Mirollo, and P.C. Matthews, Phys. Rev. Lett. 68, 2730 (1992); A. S. Pikovsky, M. G. Rosenblum, and J. Kurths, Europhys. Lett. 34, 165 (1996).

[7] D. H. Zanette and A. S. Mikhailov, Phys. Lett. A 235, 135 (1997).

[8] D. H. Zanette, Phys. Rev. E 55, 5315 (1997).

[9] D. H. Zanette and A.S. Mikhailov, Phys. Rev. E 58, 872 (1998).

[10] P. Parmananda, Phys. Rev. E 56, 1595 (1997).

[11] A. Amengual et al., Phys. Rev. Lett. 78, 4379 (1997).

[12] See M. Cross and P. Hohenberg, Rev. Mod. Phys. 65, 851 (1993), and references therein.

[13] B. Shraiman et al., Physica (Amsterdam) 57D, 241 (1992); H. Chaté, Nonlinearity 7, 185 (1994).

[14] H. Sakaguchi, Prog. Theor. Phys. 84, 792 (1990); D. Egolf and H. Greenside, Nature (London) 369, 129 (1994); R. Montagne, E. Hernández-García, and M. San Miguel, Phys. Rev. Lett. 77, 267 (1996); A. Torcini, Phys. Rev. Lett. 77, 1047 (1996). 